\title{
Modified CLIP score with the albumin-bilirubin grade retains prognostic value in HBV-related hepatocellular carcinoma patients treated with trans-catheter arterial chemoembolization therapy
}

\author{
Xiu-Rong Cai ${ }^{* 1}$, Zhan-Hong Chen ${ }^{* 1,2}{ }^{\varpi}$, Meng-Meng Liu ${ }^{* 1}$, Jin-Xiang Lin ${ }^{1}$, Xiao-Ping Zhang ${ }^{3}$, Jie Chen ${ }^{1}$, Qu \\ $\mathrm{Lin}^{1}$, Xiao-Kun $\mathrm{Ma}^{1}$, Jing-Yun Wen ${ }^{1}$, Si-Dong Xie ${ }^{4}$, Xiang-Yuan Wu ${ }^{1}$, Min Dong ${ }^{1}$ \\ 1. Department of Medical Oncology and Guangdong Key Laboratory of Liver Disease, Third Affiliated Hospital of Sun Yat-sen University, 600 Tianhe Road, \\ Guangzhou, 510630, China \\ 2. Department of Medical Oncology of Sun Yat-sen University Cancer Center, State Key Laboratory of Oncology in South China, Collaborative Innovation \\ Center for Cancer Medicine, 651 Dongfengdong Road, Guangzhou, 510060, China \\ 3. Meihua Street Community Health Service Center, Yuexiu District Guangzhou, 510000, China \\ 4. Department of Radiology and Guangdong Key Laboratory of Liver Disease, Third Affiliated Hospital of Sun Yat-sen University, 600 Tianhe Road, \\ Guangzhou, 510630, China \\ * These authors contributed equally to this work
}

$\triangle$ Corresponding authors: Zhan-Hong Chen: Address: Department of Medical Oncology and Guangdong Key, Laboratory of Liver Disease, the Third Affiliated Hospital of Sun Yat-sen University, 600 Tianhe Road, Guangzhou 510630, People's Republic of China; Tel: +86-20-85252212; E-mail: chzhanh3@mail.sysu.edu.cn. Min Dong: Address: Department of Medical Oncology and Guangdong Key, Laboratory of Liver Disease, the Third Affiliated Hospital of Sun Yat-sen University, 600 Tianhe Road, Guangzhou 510630, People's Republic of China; Tel: +86-20-85252212; E-mail: dongmin@mail.sysu.edu.cn

(0) Ivyspring International Publisher. This is an open access article distributed under the terms of the Creative Commons Attribution (CC BY-NC) license (https://creativecommons.org/licenses/by-nc/4.0/). See http://ivyspring.com/terms for full terms and conditions.

Received: 2017.09.21; Accepted: 2018.05.09; Published: 2018.06.14

\begin{abstract}
Background: The Cancer of the Liver Italian Program (CLIP) score is commonly used for prognosis prediction of hepatocellular carcinoma (HCC). The CLIP includes the Child-Pugh grade, which is relatively subjective, for hepatic encephalopathy assessment. A newly developed scoring system called albumin-bilirubin grade (ALBI grade), consists of albumin and bilirubin to assess liver function reserve objectively. Here, we substituted the ALBI grade for the Child-Pugh grade to establish the ALBI-CLIP scoring system and validated its prognostic value in hepatitis B virus (HBV)-related HCC patients treated with trans-catheter arterial chemoembolization (TACE) therapy.

Methods: We retrospectively analyzed HBV-related HCC patients who received TACE therapy. Baseline characteristics were collected and evaluated to classify patients according to ALBI-CLIP, CLIP and TNM systems. Univariate analyses using the Kaplan-Meier method and the log-rank test, as well as multivariate analysis using the Cox proportional hazards regression model, were conducted to detect independent prognostic factors for overall survival. Receiver operating characteristic (ROC) curves and a likelihood ratio test (LRT) were both utilized to compare the values of ALBI-CLIP, CLIP and TNM staging systems in predicting survival.

Results: With a total of 389 patients included in the current study, 301 (77.4\%) and $88(22.6 \%)$ were classified as Child-Pugh grade A and B, respectively. However, 152 (39.1\%), $227(58.4 \%)$ and 10 (2.5\%) patients were correspondingly classified into ALBI grade 1, 2 and 3. The areas under the curves of ALBI-CLIP, CLIP and TNM systems were $0.804,0.778$ and 0.734 , respectively, for predicting 3 -month survival; $0.796,0.778$ and 0.733 , respectively, for 6-month survival; $0.697,0.687$ and 0.644 , respectively, for 1 -year survival; and $0.618,0.612$ and 0.569 , respectively, for 2-year survival. The LRT indicated that the ALBI-CLIP and the CLIP had similar values of $\chi^{2}$ and Akaike information criterion (AIC) while the TNM system had the smallest $\chi^{2}$ value $\left(\chi^{2}=12.1,11.9,10.5 ;\right.$ AIC $=$ 2620.2, 2620.5, 2621.1 for ALBI-CLIP, CLIP and TNM, respectively).
\end{abstract}

Conclusions: In conclusion, our present study suggested that the ALBI-CLIP scoring system retained the prognostic value of the CLIP in HBV-related HCC treated with TACE therapy.

Key words: hepatocellular carcinoma; hepatitis B virus; albumin-bilirubin grade; CLIP; prognosis; transcatheter arterial chemoembolization 


\section{Introduction}

Hepatocellular carcinoma (HCC) is the second primary cause of malignancy-related mortality in males and the sixth in females, accounting for approximately 745,500 deaths worldwide [1]. Chronic hepatitis B virus (HBV) infection, which affects approximately $30 \%$ of the global population, mostly develops into liver cirrhosis and HCC, especially in China $[2,3]$. HCC in early or intermediate stages can be managed by curative hepatectomy, liver transplantation, trans-catheter arterial chemoembolization (TACE), radiofrequency ablation and radioembolization [4,5]. Though TACE is a recommended standard therapy for intermediate-stage HCC, patients beyond intermediate-stage may also receive TACE after multidisciplinary discussion. For example, BCLC-A HCC patients may receive TACE after high-risk surgery or liver transplantation. Some BCLC-C HCC patients with segmental vascular invasion may also benefit from TACE therapy. Therefore, the clinical outcomes remain heterogeneous due to different risk levels of tumor burden, liver function reserve, malignancy-related symptoms and effects of treatments [6, 7]. Furthermore, patients who can receive TACE therapy may not truly benefit from it, because TACE procedures may affect liver function. To identify a subgroup of patients who might benefit the most from TACE, a more accurate assessment system is needed.

More than ten staging systems have been defined, and some of them have been applied in clinical practice for either prognostic prediction or treatment guidelines [8]. The clinical outcomes of patients with HCC depend on tumor burden, residual hepatic function, performance status and treatment modalities. The Cancer of the Liver Italian Program (CLIP) score takes both tumor characteristics and liver function reserve into account, the latter of which is assessed by the Child-Pugh grade [9]. The CLIP scoring system is reportedly a stable and consistent prognostic model in all patients regardless of viral etiology and treatment strategies [8]. Widely applied in chronic liver diseases including HCC, the Child-Pugh grade compromises three objective parameters, including serum albumin, serum bilirubin and prothrombin time, as well as two subjective parameters, ascites and hepatic encephalopathy [10]. These two clinical factors are highly subjective due to difficulties in distinguishing different degrees of symptoms. Therefore, a newly developed scoring system called the ALBI grade, consisting of albumin and bilirubin, was proposed to objectively evaluate liver function reserve [11]. The ALBI grade has been confirmed in an international setting as a more accurate system than the Child-Pugh grade in liver function assessment of HCC [12]. Norifumi, et al. validated the value of the ALBI-TNM staging system in post-hepatectomy patients with HCC and demonstrated that this combination system significantly predicted recurrence-free survival [13]. Another previous study substituted the Child-Pugh grade for the ALBI grade in the CLIP score and found that the ALBI-CLIP retained the prognostic value of the CLIP in advanced HCC patients who received sorafenib [14].

However, to the best of our knowledge, no study has evaluated the ALBI-CLIP in the survival prediction of HCC patients who received TACE as their primary treatment. Therefore, we conducted this retrospective analysis to validate the prognostic value of the ALBI-CLIP system in HBV-related HCC patients under TACE.

\section{Methods}

\section{Patients}

We retrieved the medical records of patients who were diagnosed with HCC and hospitalized in the Third Affiliated Hospital of Sun Yat-sen University (Guangzhou, China) from July 2009 to July 2012.

Patients who met the following criteria were included: (1) over 18 years old; (2) diagnosed with HCC, which was confirmed by pathological examination or conformed to radiological criteria from the American Association for the Study of Liver Diseases [15]; (3) a history of HBV infection, which was defined as serum positive hepatitis B surface antigen (HBsAg); (4) received TACE as the primary anti-cancer treatment. Patients were excluded for: (1) viral co-infection (hepatitis C virus, human immunodeficiency virus); (2) receiving a liver resection or sorafenib after TACE therapy; (3) incomplete baseline metabolic panel data; (4) synchronal malignancies; (5) being lost to follow-up within six months.

TACE procedures were conducted under radiographic control, and clinical side-effects after TACE were all recorded. Treatment response was evaluated three or four weeks after TACE by contrast-enhanced spiral computed tomography. Patients were regularly followed up and assessed every two months, and TACE was repeated once if needed.

Written informed consents were provided by all of the patients or their family members. The clinical ethics committee of the Third Affiliated Hospital of Sun Yat-sen University (Guangzhou, China) approved all of the procedures conducted in the present study, which conformed to the Declaration of Helsinki. 


\section{Data collection}

Patients' clinical information was reviewed and acquired from the hospital database. The overall survival (OS) was set as the primary outcome of our current study and defined as the time from diagnosis to death of any cause or to the date of the last follow-up for censored patients.

Clinical demographics, pretreatment laboratory parameters (routine blood test, liver and renal biochemistry, alpha-fetoprotein (AFP), hepatitis B/C serology, etc.), and tumor-related characteristics were collected before the initial TACE therapy. Tumor-related characteristics, including the number of tumor nodules, maximum tumor diameter, portal vein tumor thrombus (PVTT) and distant metastasis, were acquired from medical imaging tests. Clinical staging of the tumors was performed in accordance with the CLIP, ALBI-CLIP and TNM staging systems. The pretreatment levels of albumin (ALB) and bilirubin (TBIL) were derived from hepatic biochemistry. Moreover, the equation for the linear value of ALBI was as follows: linear value $=\left(\log _{10}\right.$ bilirubin $\times 0.66)+($ albumin $\times-0.085)$, in which TBIL is in $\mu \mathrm{mol} / \mathrm{L}$ and ALB is in $\mathrm{g} / \mathrm{L}$. Based on the linear value, ALBI grade was then classified into three degrees: grade 1 with a value less than -2.60 , grade 2 with a value between -2.60 to -1.39 , and grade 3 with a value larger than -1.39 [12].

\section{Statistical analysis}

Chi-square test and Fisher's exact test (two-tailed) were applied to compare differences of categorical variables between groups, and the Mann-Whitney test was used for those between medians. In the univariate analyses, the Kaplan-Meier curves and the log-rank tests were conducted to estimate survival data between/among different groups. Subsequently, those significant factors were entered into a Cox proportional hazards regression model (backward method) to potentially distinguish independent prognostic factors. Hazard ratios (HR) and the $95 \%$ confidence intervals (CI) were calculated by SPSS software. To compare the values of ALBI-CLIP, CLIP and TNM staging systems in prognosis prediction, comparisons of receiver operating characteristics (ROC) curves and pairwise comparisons were applied by setting the degree of freedom at 1 . Areas under the curve (AUCs) of ROC curves were calculated and compared using the statistical method recommended by DeLong, et al. [16]. Furthermore, the likelihood ratio test (LRT) was conducted to assess the goodness of fit between the different staging systems in predicting OS. Larger $\chi^{2}$ values and smaller values of Akaike information criterion (AIC) in LRT indicated a relatively better fitting model. All statistical analyses were accomplished using SPSS (version 24.0; IBM Corp., Armonk, NY, USA), and MedCalc (version 15.8; MedCalc Software bvba, Acacialaan, Belgium). A $P$ value less than 0.05 indicated statistical significance.

\section{Results}

\section{Patient characteristics}

As shown in Table 1, a total of 389 patients with HBV-related HCC were included in our present analysis, with a median age of 52 years [Interquartile range (IQR) 44-61 years]. There were more male patients than female ones [365 vs. $24(93.8 \%$ vs. $6.2 \%)]$, which was consistent with the epidemiology of HCC. Seventy-nine $(20.3 \%)$ HCC patients were diagnosed with ascites, and 146 (37.5\%) patients received anti-viral therapies. Regarding laboratory parameters, the median serum levels of AFP, blood urea nitrogen (BUN), ALB, TBIL, alanine aminotransferase (ALT), aspartate aminotransferase (AST) and alkaline phosphatase (ALP) were $237.90 \mathrm{ng} / \mathrm{dL}, 5.05 \mathrm{mmol} / \mathrm{L}$, $38.60 \mathrm{~g} / \mathrm{L}, 0.97 \mathrm{mg} / \mathrm{dL}, 46.00 \mathrm{U} / \mathrm{L}, 55.00 \mathrm{U} / \mathrm{L}$ and $108.00 \mathrm{U} / \mathrm{L}$, respectively. In total, 301 (77.4\%) and 88 $(22.6 \%)$ patients were classified as Child-Pugh grade $\mathrm{A}$ and $\mathrm{B}$, respectively. However, according to ALBI grade, $152(39.1 \%), 227(58.4 \%)$ and $10(2.5 \%)$ patients were correspondingly divided into grade 1, 2 and 3. In subjects with a tumor condition, $250(64.2 \%)$ patients with a tumor diameter larger than $5 \mathrm{~cm}, 74(19.0 \%)$ with lymph node metastasis, 33 (8.5\%) with distant metastasis and 169 (43.4\%) with PVTT were recorded before TACE therapy. The 3-month, 6-month, 1-year, 2 -year and 5-year survival rates of patients in this study were $91.3 \%, 81.7 \%, 68.6 \%, 55.3 \%$ and $27.5 \%$, respectively.

\section{Survival analysis}

To explore the potential prognostic factors for overall survival in the current cohort of patients with HBV-related HCC after receiving TACE therapy, the OS curves were stratified by age, ascites, T category, lymph node metastasis, distant metastasis, PVTT, antiviral therapy, times of TACE, AFP, ALP, BUN, ALB and TBIL (Figure 1). As shown in the univariate analyses, AFP, T category, PVTT, distant metastasis and times of TACE were significant indicators for OS (all $P<0.05$ ) (Table 2).

Next, we conducted the Cox proportional hazards model analysis to further identify the prominently independent prognostic factors for OS. The significant indicators obtained from the univariate analyses were then entered into the Cox proportional hazards model using a backward stepwise method. As shown in Table 2, PVTT, distant metastasis and times of TACE were independent 
factors in predicting overall survival $(H R=1.887$, 1.908 and 1.470; $P<0.001,=0.003$ and 0.004 for PVTT, distant metastasis and times of TACE, respectively).

\section{Comparison of ALBI-CLIP, CLIP and TNM staging systems}

The details of the modified ALBI-CLIP score with the ALBI grade substituted for the Child-Pugh grade were listed in Supplemental Table 1. All patients were evaluated by the ALBI-CLIP and CLIP systems, both of which contained six degrees (score 0-5). Compared with their CLIP scores, a portion of patients had higher scores according to the ALBI-CLIP criteria, and the remainder had the same scores in two scoring systems (Table 3 ).

Table 1: Baseline characteristics of 389 patients with HBV-related $\mathrm{HCC}$ included in present study.

\begin{tabular}{lll}
\hline Characteristics & & No. of cases $(\%)$ \\
\hline $\begin{array}{l}\text { Total } \\
\text { Age, years (median/IQR) }\end{array}$ & & $389(100.0)$ \\
Gender & Male & $52(44-61)$ \\
& Female & $24(93.8)$ \\
Ascites & Yes & $79(20.3)$ \\
& No & $310(79.7)$ \\
Antiviral therapy & Yes & $146(37.5)$ \\
& No & $238(61.2)$ \\
& NG & $5(1.3)$ \\
Laboratory parameters (median/IQR) & \\
AFP, ng/mL & & $237.90(18.61-1000.00)$ \\
BUN, mmol/L & & $5.05(4.07-6.01)$ \\
ALB, g/L & & $38.60(34.65-42.00)$ \\
TBIL, mg/dL & & $0.97(0.71-1.46)$ \\
\hline
\end{tabular}

\begin{tabular}{|c|c|c|}
\hline \multicolumn{2}{|l|}{ Characteristics } & No. of cases (\%) \\
\hline \multicolumn{2}{|l|}{$\mathrm{ALT}, \mathrm{U} / \mathrm{L}$} & $46.00(33.00-72.00)$ \\
\hline \multicolumn{2}{|l|}{$\mathrm{AST}, \mathrm{U} / \mathrm{L}$} & $55.00(39.00-94.50)$ \\
\hline \multicolumn{2}{|l|}{ ALP, U/L } & $108.00(82.00-157.00)$ \\
\hline \multirow[t]{3}{*}{ ALBI grade } & 1 & $152(39.1)$ \\
\hline & 2 & $227(58.4)$ \\
\hline & 3 & $10(2.5)$ \\
\hline \multirow[t]{2}{*}{ Child-Pugh grade } & A & $301(77.4)$ \\
\hline & B & $88(22.6)$ \\
\hline \multirow[t]{4}{*}{ NCCN-TNM stage } & I & $21(5.4)$ \\
\hline & II & $69(17.7)$ \\
\hline & III & $120(30.8)$ \\
\hline & IV & $179(46.1)$ \\
\hline \multirow[t]{3}{*}{ Tumor size $(\mathrm{cm})$} & $\leq 5$ & $136(35.0)$ \\
\hline & $>5$ & $250(64.2)$ \\
\hline & NG & $3(0.8)$ \\
\hline \multirow[t]{2}{*}{ Lymph node metastasis } & Yes & $74(19.0)$ \\
\hline & No & $315(81.0)$ \\
\hline \multirow[t]{2}{*}{ Distant metastasis } & Yes & $33(8.5)$ \\
\hline & No & $356(91.5)$ \\
\hline \multirow[t]{3}{*}{ Portal vein tumor thrombus } & Yes & $169(43.4)$ \\
\hline & No & $219(56.3)$ \\
\hline & NG & $1(0.3)$ \\
\hline \multicolumn{2}{|l|}{ Times of TACE (median/IQR) } & $2(1-3)$ \\
\hline \multirow[t]{5}{*}{ Survival } & 3-month & $355(91.3)$ \\
\hline & 6-month & $318(81.7)$ \\
\hline & 1-year & $267(68.6)$ \\
\hline & 2-year & $215(55.3)$ \\
\hline & 5 -year & $107(27.5)$ \\
\hline
\end{tabular}

HBV: hepatitis B virus; IQR: interquartile range; ECOG: Eastern Cooperative Oncology Group; NG: Not given; AFP: alpha fetoprotein; BUN: blood urea nitrogen; ALB: albumin; TBIL: total bilirubin; ALT: alanine aminotransferase; AST: aspartate aminotransferase; ALP: alkaline phosphatase; ALBI: albumin-bilirubin grade; TNM: Tumor-node-metastasis; NCCN: The National Comprehensive Cancer Network; TACE: transcatheter arterial chemoembolization.

Table 2: Univariate and multivariate analyses of prognostic factors for overall survival in HCC patients who had undergone TACE in the present study.

\begin{tabular}{|c|c|c|c|c|c|c|c|}
\hline \multirow[t]{2}{*}{ Variable } & \multicolumn{2}{|l|}{ Univariate } & \multicolumn{5}{|c|}{ Multivariate } \\
\hline & Log-rank $\chi^{2}$ & $P$ value & $\mathrm{B}$ & SE & HR & $95 \% \mathrm{CI}$ & $P$ value \\
\hline Age, years $(>50 / \leq 50)$ & 1.885 & 0.170 & & & & & \\
\hline Ascites (yes/no) & 0.719 & 0.396 & & & & & \\
\hline \multicolumn{8}{|l|}{ Laboratory parameters } \\
\hline ALP, U/L $(>200 / \leq 200)$ & 0.952 & 0.329 & & & & & \\
\hline ALB, $(>36 / \leq 36)$ & 2.039 & 0.153 & & & & & \\
\hline $\mathrm{AFP}, \mathrm{ng} / \mathrm{dL}(>400 / \leq 400)$ & 6.492 & $0.011^{*}$ & ND & & & & \\
\hline $\mathrm{BUN}, \mathrm{mmol} / \mathrm{L}(>8.9 / \leq 8.9)$ & 3.368 & 0.066 & & & & & \\
\hline TBIL, mg/dL $(\geq 3 /<3)$ & 0.128 & 0.721 & & & & & \\
\hline TNM-T category & 12.369 & $0.006^{*}$ & & & & & \\
\hline 1 & & & $\mathrm{ND}$ & & & & \\
\hline \multicolumn{8}{|l|}{2} \\
\hline \multicolumn{8}{|l|}{3} \\
\hline \multicolumn{8}{|l|}{4} \\
\hline PVTT (yes/no) & 30.412 & $<0.001^{*}$ & 0.635 & 0.133 & 1.887 & 1.455 to 2.448 & $<0.001$ \\
\hline Lymph node metastasis (yes/no) & 0.611 & 0.435 & & & & & \\
\hline Distant metastasis (yes/no) & 13.941 & $<0.001^{*}$ & 0.646 & 0.218 & 1.908 & 1.245 to 2.927 & 0.003 \\
\hline Antivirus treatments (yes/no) & 0.202 & 0.653 & & & & & \\
\hline Times of TACE $(>2 / \leq 2)$ & 6.863 & $0.009^{*}$ & 0.385 & 0.133 & 1.470 & 1.133 to 1.905 & 0.004 \\
\hline
\end{tabular}




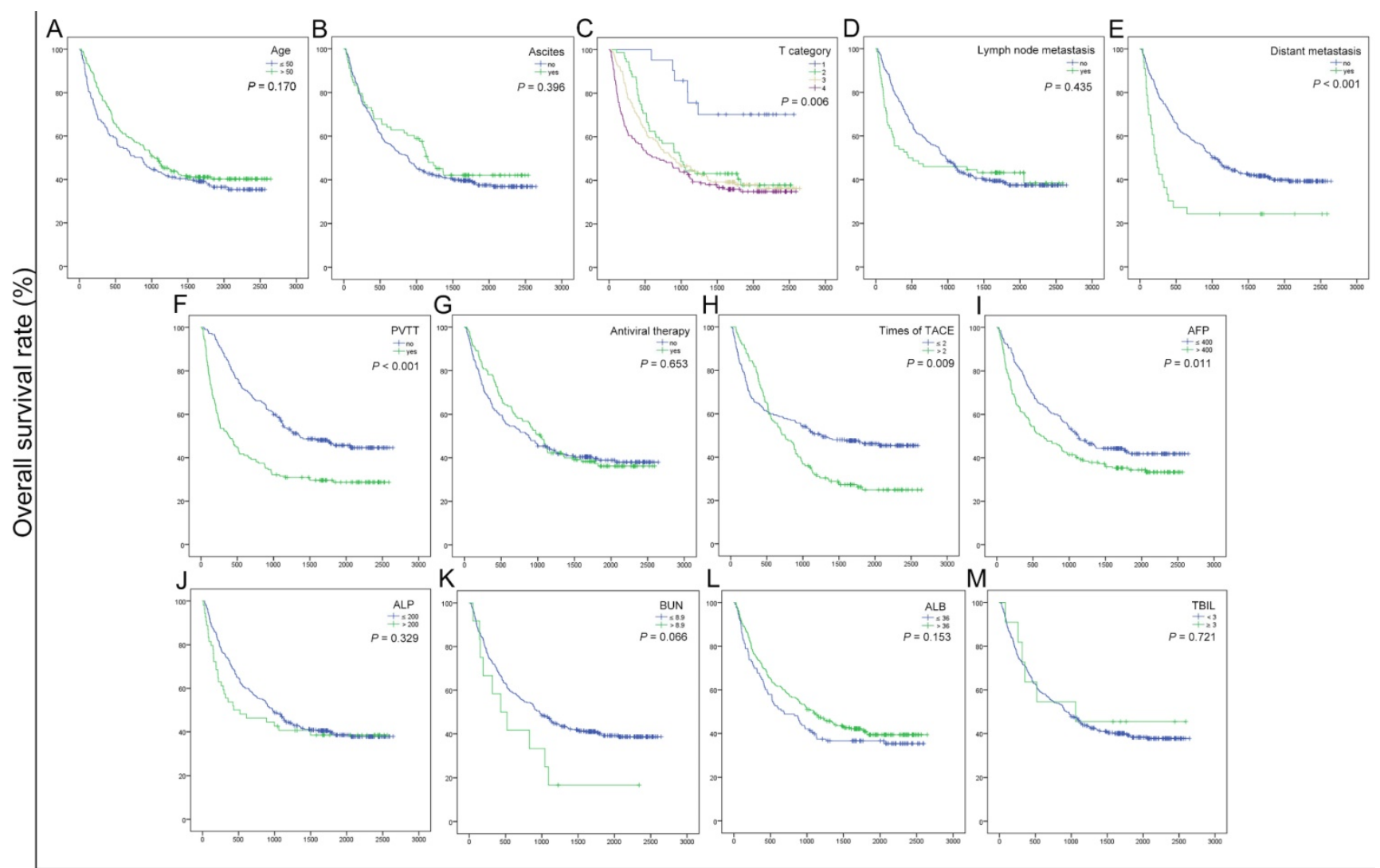

Time after diagnosis (days)

Figure 1: Kaplan-Meier curves for overall survival were stratified by different features of $389 \mathrm{HBV}$-related HCC patients treated with TACE in present study. The curves above were stratified by age (A), ascites (B), T category (C), lymph node metastasis (D), distant metastasis (E), PVTT (F), antiviral therapy (G), times of TACE $(\mathrm{H})$, AFP, ng/mL (I), ALP, U/L (J), BUN, mmol/L (K), ALB, g/L (L) and TBIL, mg/dL (M). TNM: tumor-node-metastasis; PVTT: portal vein tumor thrombus; TACE: transcatheter arterial chemoembolization; AFP: alpha fetoprotein; ALP: alkaline phosphatase; BUN: blood urea nitrogen; ALB: albumin; TBIL: total bilirubin; ALBI: albumin-bilirubin grade.

Table 3: The number of patients who were classified into different ALBI-CLIP and CLIP scores was shown below.

\begin{tabular}{lllllllll}
\hline \multicolumn{7}{c}{ ALBI-CLIP } \\
\hline CLIP & score & $\mathbf{0}$ & $\mathbf{1}$ & $\mathbf{2}$ & $\mathbf{3}$ & $\mathbf{4}$ & $\mathbf{5}$ & Total \\
& $\mathbf{0}$ & 33 & 24 & 0 & 0 & 0 & 0 & 57 \\
& $\mathbf{1}$ & 0 & 52 & 28 & 0 & 0 & 0 & 80 \\
& $\mathbf{2}$ & 0 & 0 & 45 & 42 & 0 & 0 & 87 \\
& $\mathbf{3}$ & 0 & 0 & 0 & 39 & 36 & 0 & 75 \\
& $\mathbf{4}$ & 0 & 0 & 0 & 1 & 49 & 31 & 81 \\
& $\mathbf{5}$ & 0 & 0 & 0 & 0 & 1 & 8 & 9 \\
& Total & 33 & 76 & 73 & 82 & 86 & 39 & 389
\end{tabular}

ALBI: albumin-bilirubin grade; CLIP: The Cancer of the Liver Italian Program.

The survival data of all included patients were applied in three different staging systems, including the ALBI-CLIP, CLIP and TNM systems. The Kaplan-Meier curves of these systems showed an obvious discrimination among patients in different groups (all $P<0.05$ ) (Figure 2). The median survival time of TNM stage 2, 3 and 4 was 33.0, 28.7 and 23.3 months, respectively (Figure 2-C). Visual inspection of the OS curves showed that both CLIP and ALBI-CLIP displayed a more obvious discrimination among different groups compared with the TNM system. Moreover, when we simplified patients into three groups according to their scores (score 0-1, 2-4 and 5 classified as grade 0, 1 and 2 in the ALBI-CLIP system; score $0-1,2-3$, and 4-5 classified as grade 0,1 and 2 in the CLIP system, respectively), these two systems exhibited similar differentiating ability in prognosis prediction (Figure 3). The median survival was 45.5, 24.0 and 8.6 months for simplified ALBI-CLIP grade 0 , 1 and 2, respectively, and 38.4, 29.3 and 8.8 months for simplified CLIP grade 0,1 and 2, respectively.

To primarily compare the abilities of the ALBI-CLIP, CLIP and TNM systems in prognosis prediction, their ROC curves were compared. The AUCs of the ALBI-CLIP, CLIP and TNM systems were $0.804,0.778$ and 0.734 , respectively, for predicting 3-month survival; 0.796, 0.778 and 0.733, respectively, for 6-month survival; $0.697,0.687$ and 0.644, respectively, for 1-year survival; and 0.618, 0.612 and 0.569 , respectively, for 2 -year survival. The above indicated that the ALBI-CLIP and the CLIP had similar AUCs, both of which were larger than that of the TNM system (Figure 4). Furthermore, pairwise comparison of ROC curves showed that no significant difference was observed between the ALBI-CLIP and the CLIP in predicting 3-month, 6-month, 1-year and 2-year survivals (all $P>0.05$ ) (Supplemental Table 2). 

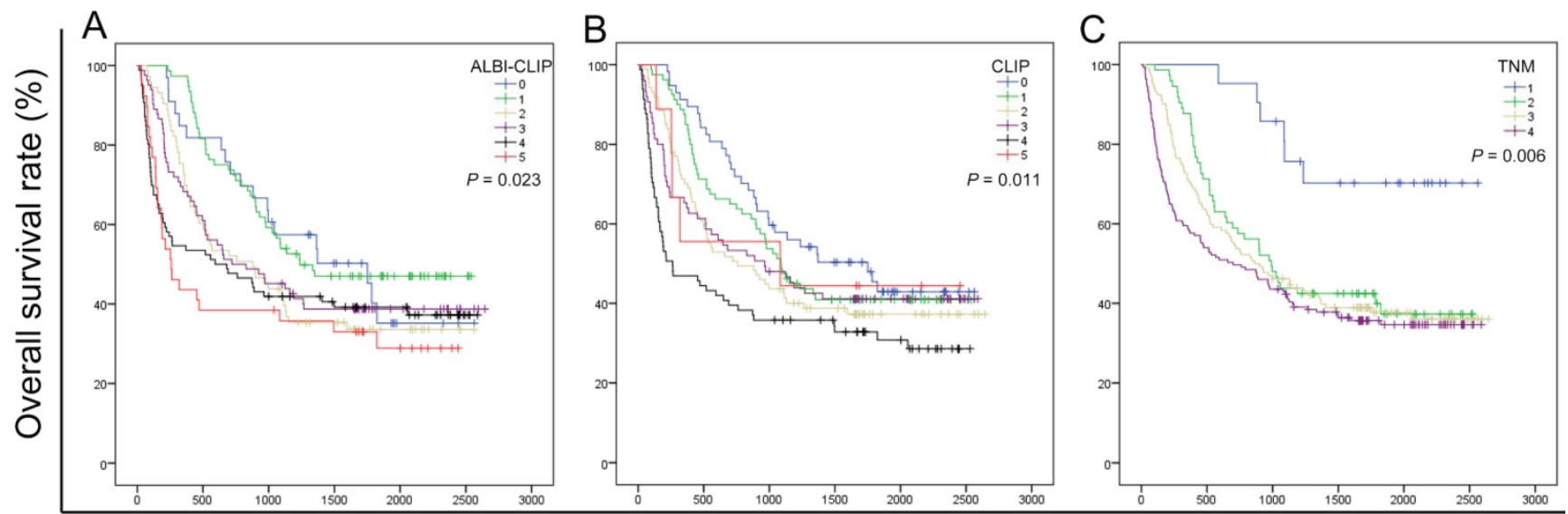

Time after diagnosis (days)

Figure 2: Kaplan-Meier curves for overall survival of $389 \mathrm{HBV}$-related HCC patients who have undergone TACE in present study, which were stratified by the ALBI-CLIP (A), CLIP (B), and TNM (C) staging systems. OS was significantly different among subgroups stratified by the above variables (all $P<0.05)$. ALBI: albumin-bilirubin grade; CLIP: The Cancer of the Liver Italian Program; TNM: tumor-node-metastasis.
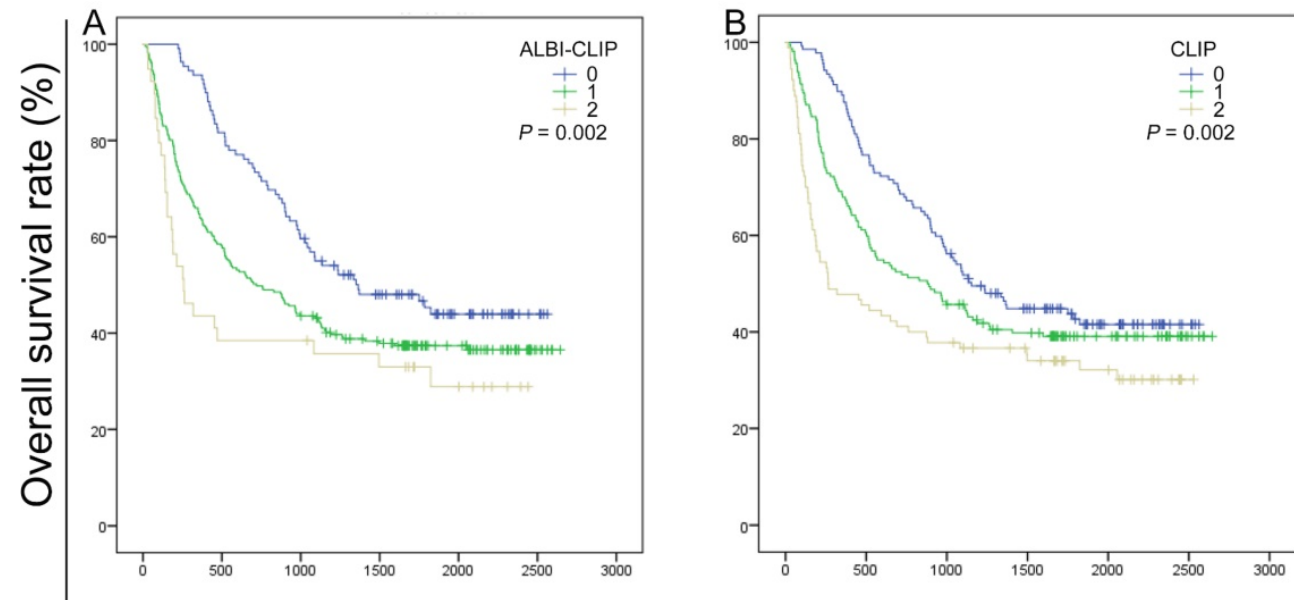

Time after diagnosis (days)

Figure 3: Kaplan-Meier curves for overall survival of 389 patients who were diagnosed with HBV-related HCC and have undergone TACE in present study. Survival curves were stratified into 3 grades by the ALBI-CLIP (A) and CLIP (B) scoring systems. ALBI: albumin-bilirubin grade; CLIP: The Cancer of the Liver Italian Program.
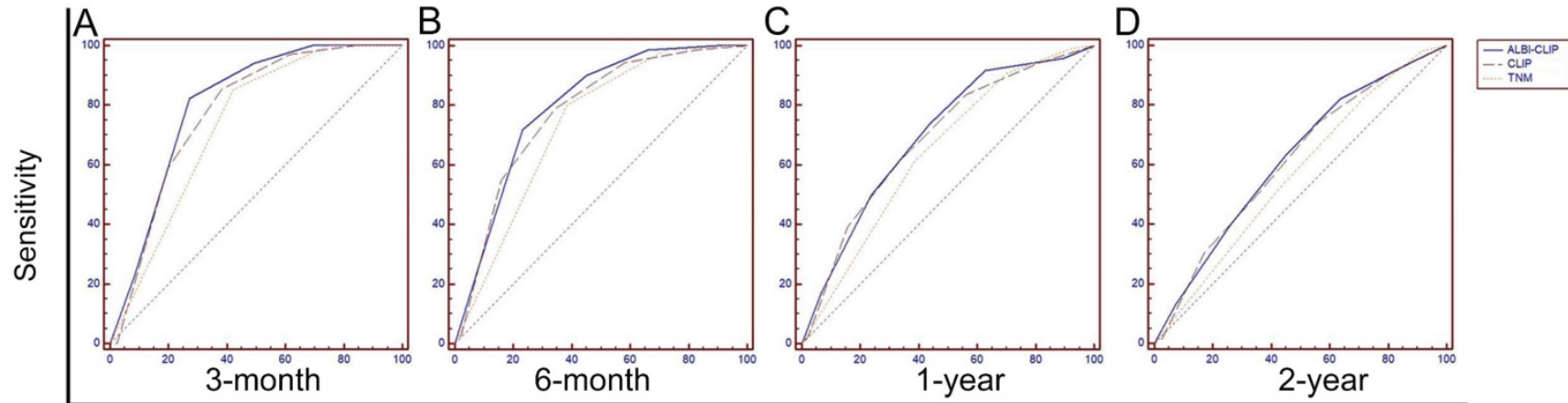

100-Specificity

Figure 4: Comparisons of the area under curve (AUC) for overall survival prediction among the ALBI-CLIP, CLIP and TNM staging systems using ROC curves. The comparisons were conducted for 3-month survival (A), 6-month survival (B), 1-year survival (C), and 2-year survival (D). ALBI: albumin-bilirubin grade; CLIP: The Cancer of the Liver Italian Program; TNM: tumor-node-metastasis.

Moreover, the LRT indicated larger $\chi^{2}$ values of the ALBI-CLIP and the CLIP compared with that of TNM system, but similar values of AIC $\left(\chi^{2}=12.1\right.$, 11.9, 10.5; $\mathrm{AIC}=2620.2,2620.5,2621.1$ for ALBI-CLIP,
CLIP and TNM, respectively) (Table 4), further suggesting that the ALBI-CLIP retains the predictive value of the CLIP in HBV-related HCC after TACE treatments. 
Table 4: Comparisons of the ALBI-CLIP and CLIP staging systems in the values of prognosis prediction for overall survival of patients in present study.

\begin{tabular}{llll}
\hline Staging system & Likelihood ratio test & AIC & $P$ value \\
& $\chi^{2}$ & & \\
\hline ALBI-CLIP & 12.1 & 2620.2 & 0.001 \\
CLIP & 11.9 & 2620.5 & 0.001 \\
TNM & 10.5 & 2621.1 & 0.001 \\
\hline
\end{tabular}

ALBI: albumin-bilirubin grade; CLIP: The Cancer of the Liver Italian Program; AIC: Akaike information criterion.

\section{Discussion}

The ALBI-CLIP has been assessed in HCC patients receiving antiangiogenic drugs, but not in those treated with TACE. Our current study suggested that the ALBI grade, an objective and simple assessment of hepatic function, could be a substitute for the Child-Pugh grade to be integrated into the CLIP system to predict the prognosis of HBV-related HCC patients under TACE treatments.

Cheng AL, et al. evaluated the prognostication of the ALBI-CLIP among 142 patients with advanced HCC undergoing first-line antiangiogenic therapy and concluded that the ALBI-CLIP retained the prognostic value of the CLIP in advanced HCC patients who received sorafenib [14]. However, their cohort was small. Salem $\mathrm{R}$, et al. assessed the validity of the ALBI grade as a predictor of survival in 765 HCC patients undergoing TACE. They concluded that the ALBI grade outperforms the Child-Pugh class in discriminating survival of patients treated with TACE or yttrium-90 (Y) radioembolization [17]. However, the ALBI-CLIP and the CLIP systems were not comparably assessed in this study. Stephen L Chan, et al. evaluated the prognostic performance of eleven staging systems in 1,973 Chinese patients with HCC and concluded that the ALBI-CLIP and the CLIP are two of the most accurate prognostic models among current staging systems [18]. However, the authors did not compare the prognostic value of the ALBI-CLIP with the CLIP alone in patients receiving TACE. As far as we know, no study has focused on the assessment of the predictive value of the ALBI-CLIP in HBV-related HCC patients receiving TACE therapy so far. Therefore, we aimed to validate the prognostic value of the ALBI-CLIP in this group of patients.

We included HBV-related HCC patients who had received TACE therapy as their primary anti-cancer treatments. After univariate and multivariate analyses of medical data, we found that PVTT, distant metastasis and times of TACE were independent prognostic factors for this cohort of patients.

Both visual inspection and formal statistical analyses indicated that the discriminating ability of the ALBI-CLIP was as good as the CLIP system. When we simplified both ALBI-CLIP scores and CLIP scores into 3 categories, the median survivals were similar between their corresponding grades, suggesting that the ALBI-CLIP retained the prognostic value of the CLIP among patients in our study. Median survivals of HBV-relative HCC patients after TACE in either simplified ALBI-CLIP grade 3 or simplified CLIP grade 3 were less than 9 months, indicating that this cohort of patients might benefit less from TACE therapy. Therefore, for a group with relatively unfavorable prognosis, other treatment options, such as multikinase inhibitors sorafenib or regorafenib, immune checkpoint inhibitors should be considered.

Though TACE therapy is recommended as first-line treatment for intermediate-stage HCC, the median survival of this population ranges from 14 to 45 months, which might due to the heterogeneity of tumor burden, residual hepatic function and performance status of these patients [19, 20]. Our current analysis indicated that cancer-related characteristics, including PVTT and distant metastasis, as well as times of TACE were independent risk factors for HBV-related HCC undergoing TACE treatments. Alternatively, TACE treatments might have deteriorating effects on liver function, especially for patients with liver cirrhosis. Patients who underwent TACE treatments may not respond or even have liver function failures after the procedures. Therefore, repeated assessments of liver function and therapeutic response are necessary and important in managing HBV-relative HCC. The Child-Pugh grade is commonly used in clinical practice to evaluate hepatic function reserve. However, clinical assessment of both ascites and hepatic encephalopathy is subjective, leading to interobserver variation. In contrast, the ALBI grade was composed of only serum levels of ALB and TBIL derived from hepatic biochemistry tests. Furthermore, the ALBI grade was confirmed in HCC patients at any stage in an international setting. It was reported to perform as well as the Child-Pugh grade with objectivity and ease of operation [12]. The ALBI accurately evaluated the liver function reserve of both Eastern and Western HCC patients across each BCLC stage, including those who received TACE [21]. The CLIP score, mostly applied in prognosis prediction rather than to aid in decision-making, has been externally validated in clinical practice [22]. One of our previous studies found that the CLIP system performed best among seven staging systems in predicting prognosis of patients diagnosed with HBV-related HCC [23]. Other researchers also found that the CLIP system was the best staging system in prognostic prediction of 3,182 patients with HCC 
comparing with other ten staging systems [8]. Therefore, we did not carry out comprehensive comparisons of the CLIP with other scoring systems, but only conducted comparisons of the ALBI-CLIP, CLIP and TNM systems in our study.

Several limitations still exist in our current study. First, as a retrospective study, selection bias was difficult to avoid though both inclusion and exclusion criteria were strictly conducted. Second, the total number of patients enrolled in this study was relatively small, and all cases were from one single center. Third, a lack of validation cohorts limited the further confirmation of the ALBI-CLIP score in HBV-related HCC patients after TACE therapy. Lastly, all included patients were diagnosed with HBV-related HCC, which limited our results being expanded to HCV-related and other types of HCC.

In conclusion, our present study suggested that the ALBI-CLIP scoring system retained the prognostic value of the CLIP score in HBV-related hepatocellular carcinoma treated with TACE therapy.

\section{Abbreviations}

HBV: hepatitis B virus; HCC: hepatocellular carcinoma; AFP: alpha fetoprotein; BUN: blood urea nitrogen; ALB: albumin; TBIL: total bilirubin; ALT: alanine aminotransferase; AST: aspartate aminotransferase; ALP: alkaline phosphatase; ALBI: albumin-bilirubin grade; PVTT: portal vein tumor thrombus; TNM: Tumor-node-metastasis; NCCN: The National Comprehensive Cancer Network; TACE: transcatheter arterial chemoembolization; OS: overall survival; ROC curve: receiver operating characteristics curve.

\section{Acknowledgements}

This study was supported by grants from the National Natural Science Foundation of China (grant no. 81502271, 31600710 and 81372374), the Combination Project of Production, the Scientific Research Project for University belong to Guangzhou Municipal (grant no. 1201630019), Natural Science Foundation of Guangdong (grant no. 2014A03 0313146, 2016A030313302 and 2017A030313537), the Project on the Integration of Industry, Education and Research of Guangdong Province (grant no. 2012B0 91100460), Guangzhou Science and Technology Plan (201709010030) and Medical Scientific Research Foundation of Guangdong Province (No: A2015109).

\section{Supplementary Material}

Supplementary tables.

http://www.jcancer.org/v09p2380s1.pdf

\section{Competing Interests}

The authors have declared that no competing interest exists.

\section{References}

1. Torre LA, Bray F, Siegel RL, Ferlay J, Lortet-Tieulent J, Jemal A. Global cancer statistics, 2012. CA: a cancer journal for clinicians. 2015; 65: 87-108.

2. Trepo C, Chan HL, Lok A. Hepatitis B virus infection. Lancet. 2014; 384: 2053-63.

3. Liu J, Zhang S, Wang $Q$, Shen $H$, Zhang $M$, Zhang $Y$, et al. Seroepidemiology of hepatitis $B$ virus infection in 2 million men aged 21-49 years in rural China: a population-based, cross-sectional study. The Lancet Infectious diseases. 2016; 16: 80-6.

4. Bruix J, Gores GJ, Mazzaferro V. Hepatocellular carcinoma: clinical frontiers and perspectives. Gut. 2014; 63: 844-55.

5. Bruix J, Sherman M, American Association for the Study of Liver D. Management of hepatocellular carcinoma: an update. Hepatology (Baltimore, Md). 2011; 53: 1020-2.

6. Lencioni R. Loco-regional treatment of hepatocellular carcinoma in the era of molecular targeted therapies. Oncology. 2010; 78 Suppl 1: 107-12.

7. Forner A, Gilabert M, Bruix J, Raoul JL. Treatment of intermediate-stage hepatocellular carcinoma. Nature reviews Clinical oncology. 2014; 11: 525-35.

8. Liu PH, Hsu CY, Hsia CY, Lee YH, Su CW, Huang YH, et al. Prognosis of hepatocellular carcinoma: Assessment of eleven staging systems. Journal of hepatology. 2016; 64: 601-8.

9. investigators TCotLIPC. A new prognostic system for hepatocellular carcinoma: a retrospective study of 435 patients. Hepatology (Baltimore, Md); 1998; 28(3): 751-5.

10. Shetty K, Rybicki L, Carey WD. The Child-Pugh classification as a prognostic indicator for survival in primary sclerosing cholangitis. Hepatology (Baltimore, Md). 1997; 25: 1049-53.

11. Hiraoka A, Kumada T, Kudo M, Hirooka M, Tsuji K, Itobayashi E, et al. Albumin-Bilirubin (ALBI) Grade as Part of the Evidence-Based Clinical Practice Guideline for HCC of the Japan Society of Hepatology: A Comparison with the Liver Damage and Child-Pugh Classifications. Liver Cancer. 2017; 6: 204-15.

12. Johnson PJ, Berhane S, Kagebayashi C, Satomura S, Teng M, Reeves HL, et al. Assessment of liver function in patients with hepatocellular carcinoma: a new evidence-based approach-the ALBI grade. Journal of clinical oncology : official journal of the American Society of Clinical Oncology. 2015; 33: 550-8.

13. Harimoto N, Yoshizumi T, Sakata K, Nagatsu A, Motomura T, Itoh S, et al. Prognostic significance of combined albumin-bilirubin and tumor-node-metastasis staging system in patients who underwent hepatic resection for hepatocellular carcinoma. Hepatology research : the official journal of the Japan Society of Hepatology. 2017; 47(12): 1289-98.

14. Shao YY, Liu TH, Lee YH, Hsu CH, Cheng AL. Modified CLIP with objective liver reserve assessment retains prognosis prediction for patients with advanced hepatocellular carcinoma. Journal of gastroenterology and hepatology. 2016; 31: 1336-41.

15. Bruix J, Sherman M, Practice Guidelines Committee AAftSoLD. Management of hepatocellular carcinoma. Hepatology (Baltimore, Md). 2005; 42: 1208-36.

16. DeLong ER, DeLong DM, Clarke-Pearson DL. Comparing the areas under two or more correlated receiver operating characteristic curves: a nonparametric approach. Biometrics. 1988; 44: 837-45.

17. Hickey R, Mouli S, Kulik L, Desai K, Thornburg B, Ganger D, et al. Independent Analysis of Albumin-Bilirubin Grade in a 765-Patient Cohort Treated with Transarterial Locoregional Therapy for Hepatocellular Carcinoma. Journal of vascular and interventional radiology: JVIR. 2016; 27: 795-802.

18. Chan AW, Chong CC, Mo FK, Wong J, Yeo W, Johnson PJ, et al. Incorporating albumin-bilirubin grade into the cancer of the liver Italian program system for hepatocellular carcinoma. Journal of gastroenterology and hepatology. 2017; 32: 221-8.

19. Omata M, Cheng AL, Kokudo N, Kudo M, Lee JM, Jia J, et al. Asia-Pacific clinical practice guidelines on the management of hepatocellular carcinoma: a 2017 update. Hepatol Int. 2017; 11: 317-70.

20. Llovet JM, Bruix J. Novel advancements in the management of hepatocellular carcinoma in 2008. Journal of hepatology. 2008; $48 \mathrm{Suppl}$ 1: S20-37.

21. Pinato DJ, Sharma R, Allara E, Yen C, Arizumi T, Kubota K, et al. The ALBI grade provides objective hepatic reserve estimation across each BCLC stage of hepatocellular carcinoma. Journal of hepatology. 2017; 66: 338-46. 
22. Urakawa H, Kora SI, Mitsufuji T, Osame A, Higahsihara H, Yoshimitsu $\mathrm{K}$. Which system is better to predict prognosis of patients with hepatocellular carcinoma treated by transcatheter arterial chemoembolization as initial therapy? Comparison between CLIP and JIS in a Japanese population. Acta radiologica. 2016; 57: 1445-52.

23. Chen ZH, Hong YF, Lin J, Li X, Wu DH, Wen JY, et al. Validation and ranking of seven staging systems of hepatocellular carcinoma. Oncol Lett. 2017; 14: 705-14. 